\title{
Bisensory memory in normal and reading disability children
}

\author{
M. SUSANNE DAVIS and NORMAN W. BRAY \\ University of Cincinnati, Cincinnati, Ohio 45221 \\ and \\ Cincinnati Center for Developmental Disorders \\ 3300 Elland Avenue, Cincinnati, Ohio 45229
}

\begin{abstract}
Normal and reading disability children were tested for memory of item and order information in a sequential memory task with auditory and visual stimuli. There were three auditory-visual digit pairs on each trial, with a 1-sec delay between successive pairs. Either one auditory-visual pair (pair condition) or two digits from one modality (modality condition) were tested. More errors were made in the pair than in the modality condition on a measure of item information but not on a measure of order information. Reading disability children made more errors than normal children, but there were no Reading Group by Condition interactions. These results suggest that: (a) the reading groups differed on general information-processing capabilities, but (b) both reading groups have the same degree of difficulty with organization of temporal-modality information.
\end{abstract}

Bisensory memory for auditory and visual information is an important component of reading comprehension (Silverston \& Deichmann, 1975). A procedure for studying bisensory memory in normal and reading disability children has been developed in a series of studies by Senf (Senf, 1969; Senf \& Freundl, 1971). In this task, one digit is presented to the eye simultaneously with a different digit to the ear. After three auditory-visual pairs, the child attempts to recall the six digits in one of two conditions: (a) a pair condition in which the digits are recalled as auditory-visual pairs in order of presentation, beginning with the first auditory digit, or (b) a modality condition in which the digits presented aurally are recalled in sequence followed by the visually presented digits.

In both pair and modality conditions, Senf found fewer errors for young normal than for young reading disability children, but the difference between reading groups was larger in the modality than in the pair condition. Although Senf and Freundl (1971) did not offer a full explanation of the reading group difference on pair recall, they did suggest that the difference between reading groups in the modality

This paper is based on a Master's thesis submitted to the Department of Psychology. University of Cincinnati, by the first author. The authors wish to thank Joel S. Warm, who sponsors this paper and takes full editorial responsibility for it. The cooperation of the principals, teachers, and students of Struble, Pleasant Run, and Monfort Heights elementary schools is gratefully acknowledged. This project was supported by Grant Nos. 912 and 918, awarded by the Bureau of Community Health Services, Health Services Administration and by Public Health Service, Grant No. 59-25297/5-02, awarded by Region V, Social and Rehabilitation Service, DHEW. Requests for reprints should be sent to Norman W. Bray, Psychology Department, Cincinnati Center for Developmental Disorders, 3300 Elland Avenue, Cincinnati, Ohio 45229. condition was due to the manner in which temporal and modality information must be organized in memory. According to their explanation. the items must be divided into auditory and visual sets and, in forming the two sets, the temporal order of items within a modality must be derived from the three successive auditory-visual pairs. At recall, the two sets must be organized by temporal order of the items within each modality. Reading disability children appear to have more difficulty than normal children with deriving ordered modality information from auditory-visual pairs and in utilizing this ordered information at recall.

While Senf and Freundl stress the importance of temporally ordered information in explaining the difference between reading groups on modality recall. recent studies (Bjork \& Healy. 1974; Healy, 1974) have suggested that in similar tasks, information about particular items is stored independently of information about the temporal order of the items. Senf (1969) and Senf and Freundl (1971) did not design their studies to investigate the differences between item and order information but they used two measures related to the question. A gross error measure provided some data concerning retention of item information since the order in which the items were recalled was ignored. If $\mathrm{g}=$ number of gross errors (omissions + intrusions) and $n=$ total number of items tested, then the proportion of gross errors was $\mathrm{g} / \mathrm{n}$. An order error measure provided an index of overall level of accuracy for ordered information. If $i$ $=$ number of items recalled in the incorrect order, then the proportion of order errors may be represented as $\mathrm{i} / \mathrm{n}$.

Since the general pattern of results described previously was found for both the gross and the order error measures, it would appear that the difficulties 
reading disability children have with the organization of temporal and modality information influences the retention of both item and order information. However, a closer analysis suggests two potential problems with this interpretation. First, since all items were tested on each trial, it is possible that both measures were contaminated by interference from multiple recall attempts (Kintsch, 1970) and that the reading group difference on modality recall was due to greater output interference for the reading disability children. In the present study, a recall probe was used to test only two of the digits on each trial. This procedure minimized the possibility of output interference and therefore allowed a less ambiguous interpretation of the measures. Second, the order error measure, $i / n$, is influenced by the number of items either intruded or omitted from recall, and the difference between reading groups on modality recall may be due to differential intrusion and omission rates. Healy (1974) has suggested that a clearer index of retention of order information is a conditional proportion based on only those items recalled. In the present study, the conditional proportion of order errors was $\mathrm{i} /(\mathrm{n}-\mathrm{g})$. Providing $\mathrm{g} / \mathrm{n}$ does not approach 1.0 , the conditional measure will not be influenced by the number of items either intruded or omitted.

\section{METHOD}

\section{Subjects}

The subjects were 24 reading disability and 24 normal children from classes at three predominantly middle-class schools in Hamilton County, Ohio. The children were between the ages of 7.0 and 10.5 years and of normal intelligence as measured by either the Otis Quick Intelligence Test, the Stanford-Binet Intelligence Scale, or the Wechsler Intelligence Scale for Children. All children were in their age-appropriate grade placements.

The reading disability subjects were children who scored below the 45th percentile on the Paragraph Meaning subtest of the Stanford Achievement Test $(M=23.4, S D=11.7)$, and the norma subjects were children scoring above the 55th percentile on this subtest $(M=78.5, S D=8.1)$.

\section{Experimental Design}

The design was a 2 by 2 by 2 mixed factorial with two between-subjects variables and one within-subjects variable. Reading group (normal and reading disability) and probe type (pair and modality) were the between-subjects factors, and mode of presentation (auditory and visual) was the within-subjects factor.

\footnotetext{
Apparatus and Stimulus Materials

Auditory and visual stimuli were presented simultaneously by a Bell and Howell language master (Model No. 711B). Visual stimuli appeared on the language master cards in .64-cm black block print. A wooden screen was placed between the subject and the stimulus card so that visual stimuli appeared one at a time through a $2.54-\mathrm{cm}$-square window centered in the screen. The visual stimuli moved across the window from the subject's left to his right, with a 1 -sec interval between the onset of successive pairs. The exposure duration for each visual item was $.43 \mathrm{sec}$. The auditory stimuli, recorded in a slow clear female voice, were synchronized with the visual stimuli so that the onset of the recording of a digit and the onset of a visual stimulus were simultaneous. The duration for each auditory item was approximately $.50 \mathrm{sec}$.
}

Digits for each trial were selected from numbers 1 through 9 without replacement. All digits occurred 4 times in 8 trials of a pretraining phase and 16 times in 24 trials of the experimental phase. In the experimental trials, each digit occurred eight times in the auditory and eight times in the visual modality.

\section{Procedure}

All subjects were tested individually in a small quiet room. The testing session required approximately $20 \mathrm{~min}$. The subject was seated directly in front of the apparatus with the window for the visual stimuli at eye level. Each subject was instructed to remember all the numbers he would see and hear on each trial. There were eight trials of pretraining in which the number of pairs was increased from one to three, followed by 24 experimental trials with three pairs each.

After each sequence of digits, a probe sheet was presented to cue recall. The probe sheet contained a 3 by 2 grid composed of two rows of three adjacent $2 \times 2 \mathrm{~mm}$ boxes. These six boxes represented the six stimuli (three pairs of two digits each), the top row corresponding to the three visual items, the bottom row to the three auditory items. On each sheet, the word SAW was typed to the left of the top row and HEARD to the left of the bottom row. Question marks in two of the six boxes indicated the two digits to be reported. On each trial in the pair condition, one of three auditory-visual pair locations was tested (the first, second, or third auditory-visual pair). On each trial in the modality condition, one of six pair locations was tested (the first two visual, last two visual, first and last visual; first two auditory, last two auditory, or first and last auditory digits). The pair locations tested occurred randomly throughout the 24 experimental trials, with each pair location occurring eight times in the pair condition and each modality location occurring four times in the modality condition. In the modality condition, there were 12 visual modality tests and 12 auditory tests.

\section{RESULTS}

\section{Item Errors}

An item error consisted of any digit that was omitted or intruded. The proportion of item errors for a subject was the total number of item errors divided by the total number of digits tested (24), with auditory and visual modes computed separately.

An analysis of variance of these data resulted in a significant effect due to reading group, $F(1,44)=$ $7.32, \mathrm{p}<.01$, the means being .42 for the reading disability and .30 for the normal children. The pair condition produced significantly more item errors than the modality probe type, $F(1,44)=18.70$, $\mathrm{p}<.001$, with means of .46 and .26 , respectively. Finally, the main effect of mode of presentation was signiticant, $F(1,44)=27.02, p<.001$, with means of .44 errors for the visual and .28 for auditory. There were no significant interactions ( $p>.10$ in all cases).

\section{Order Errors}

An order error consisted of any digit that was recalled but not reported in the correct order. The proportion of order errors for a subject was the number of order errors divided by the total number of digits recalled correctly regardless of order, with scores for the auditory and visual modalities computed separately.

An analysis of variance of these data indicated a significant main effect for reading group, $F(1,44)=$ $4.82, \mathrm{p}<.01$, the means being .23 for the reading 
disability and .13 for the normal children. A significant difference due to mode of presentation was also found, $F(1,44)=15.26, p<.001$, with visual and auditory means of .25 and .11 , respectively. The means for the pair and modality recall conditions were .18 and .19 , respectively, which was not a significant difference $(F<1)$. There were no significant interactions $(\mathrm{p}>.25$ in all cases).

\section{DISCUSSION}

As in previous investigations (e.g., Senf, 1969; Senf \& Freundl, 1971), reading disability children made more errors than normal children on both item and order information measures. Unlike previous investigations, there was no interaction between reading group and recall condition on either measure. This pattern of results does not support the hypothesis that reading disability children have more difficulty than normals with organization of temporal and modality information. Since the probe procedure used here minimized interference due to output (Kintsch, 1970), it may be that the previous findings of an interaction between reading group and recall condition were due to greater output interference in the reading disability group.

The finding of a reading group difference for pair and modality recall on both the order and the item error measures may indicate a difference in general information processing capabilities. However, the lack of a difference between pair-modality conditions for both reading groups on the order error measure suggests that the same amount of order information is lost for pair and modality recall in both reading groups. This finding points out the importance of eliminating intrusions and omissions in calculating the amount of order information retained.

The use of the probe procedure and the conditional order error measure evidently did not influence other basic findings of the present study. As is generally the case in investigations of bisensory memory (Calfee, Chapman, \& Venesky, 1972), reading disability children performed more poorly than normal children and there were fewer errors for auditory than for visual items. The fact that these findings replicate effects in previous studies lends credence to the unique results obtained here. It is recognized, of course, that further investigation of auditoryvisual information processing in normal and reading disability children is necessary. The present results indicate that this research can profitably focus on interference effects in shortterm retention of ordered information.

\section{REFERENCES}

BJork, E. L., \& HeAly, A. F. Short-term order and item retention. Journal of Verbal Learning and Verbal Behavior, 1974. 13. 80-97.

Calfee, R., Chapman, R., \& Venesky, R. How a child needs to think to learn to read. In L. W. Gregg (Ed.), Cognition in learning and memory. New York: Wiley, 1972.

HEALY, A. F. Separating item from order information in short-term memory. Journal of Verbal Learning and Verbal Behavior, 1974. 13. 644-655.

Kintsch, W. Learning, memory, and conceptual processes. New York: Wiley, 1970.

SENF, G. M. Development of immediate memory for bisensory stimuli in normal children and child ren with learning disabilities. Developmental Psychology Monograph, 1969, 1 (6, part 2), 1-28.

Senf, G. M., \& Freunde, P. C. Memory and attention factors in specitic learning disabilities. Journal of Learning Disabilities. $1971,4,36-48$.

Silverston, R. A., \& Deichmann, J. W. Sense modality research and the acquisition of reading skills. Review of Educational Research, 1975, 45, 149-172.

(Received for publication August 8, 1975.) 